Rev. Chil. Pediatr. 68 (2); 99-103, 1997

\title{
Movimientos anormales y encefalitis
}

\author{
Raúl Escobar H. '; Francisco Mena C. ${ }^{1}$; Ricardo Erazo T. '
}

\begin{abstract}
Resumen
Los movitn e-tos anormales no convulsivos, secundarios a encefaliris; si bien son poco frectuentes, representan un importanie desctio para diagnóstico diferencial, tratamiento y pronóstico de estos enfermos, como se ilustra en cinco cosos cliricos cuyas edades flucruaban ente 2 y 10 años. Todos sufrian intensos rovimientos onormales y la explicación más probable parc su enfermedad, en bose a os demás hechos clínicos, las alıerociones electroencefalográficas y los exórrenes de imogen fue encetalitis. En la evolucićn de la fase aguda de su rastorno predominabon severos rovimienros extrapiramidales El seguimento a largo plazo mastró que, si bien las manifestaciones iniciales pueden ser muy imporiantes, el pronóstico a mediano y lorgo plazo de los pacientes cuya encefalitis se expresa como mcvimientos anormales fue bueno vo que los pacien'es. con sólo una excepción-atribuble $\mathrm{c}$ un ep sodio de hipoxo severa ocurrido variass semanas después y relacionado con asistencia mecánica de la ventilación por ne.jmo-ía severo-, no mostraban secuelas a largo plazo.
\end{abstract}

\{Palabras clave: encefalitis, mov mienlos cnorroles.;

\section{Abnormal movements and encephalitis}

Non convulsive abnomal movements associared with encephalitis, although infrequent, are of greal impertance becouse of the difficulies that they posse to diagnosis, treament and prognosis of affecled patients. The cinical cases of five patients aged 2 lo 10 years whose final diognosis was encepholitis and their predominant symptoms were severe non convulsive extropiramidal abno'nal movements ore described. At iong lerm follow-up all abnormal movements dissapeored and there were no neurologicol sequalae in four of these patients. The only ins.ance of severe cenlral nervcus sysiem sequelae was ieloted to a significanl hicoxic accident during assisted mechanical ventlalion in the cou'se s: bilaleral pneumonia and sepsis, several weeks after hospital discharge from the primary neurological disecse, in a seven years old girl whose residual -eviological abrormalilies did not show further increase from that lime

[Key words: encepnalits, movement disorders.)

Las alteraciones del movimiento suelen ser atribuidas a anormalidades del sistema nervioso central (SNC), particularmente de los núcleos (ganglios) basales y sus estructuras de conexion. Una serie de afecciones (tóxicas, metabólicas, degenerativas), se han relacionado con este tipo de anomalias del movimiento, sin embargo, su

1. Servicio de Neurologia y Psiquiatría. Hospital Luis Calvo Mackenna. Escuela de Medicina, Universidad de Chile. etiología no ha sido determinada con precision. Las infecciones del SNC, incluyendo meningitis purulentas $y$ encefalitis, también han sido involucrados en su patogenia ${ }^{1.4}$. Los movimientos anormales secundarios a encefalitis, si bien son de ocurrencia esporádica, constituyen un desafío para el diagnóstico diferencial, el tratamiento y particularmente el pronóstico. El motivo de esta comunicación es describir los casos de cinco pacientes, con severos movimientos anormales, en los cuales se propuso el diagnóstico de encefalitis como explicación de sus trastornos. 


\section{Casos Clínicos}

1. Niño de 5 años de edad, previamente sano. Sin antecedentes de ingestión de fártnacos o exposición a tóxicos. Su enfermedad comenzo con irritabilidad y movimientos coreicos leves $y$ focales, que luego de una semana se generalizaron e intensificacon, agregándose además temblor intencional de extremidades. Hospitalizado en otro centro por cinco dízs. se diagnosticó corea menor (Sydenham). Después del alta evolucionu con labilidad emocional, insomnio pertinaz $y$, posteriormente, intensos inovimientos coreoaletósicos de tronco y extremidades, temblor generalizado y movimientos distónicos faciales y orolinguales. Hospitalizado nuevamente, ahora por seis semanas, evolucionó inicialmente con disminuciones y exacerbaciones fuctuantes de sus síntomas. pero finalmente éstos disminuyeron progresivaunente. Los siguientes exámenes dieron resultados negativos a normales: examen citoquímico $y$ microbiológico de líquido cefatortaquídeo (LCR) en la primera y segunda semana de la segunda hospitalización: tomografía axial computadorizada (TAC) y resonancia magnética (RM) de cerebro; cultivos de deposiciones; pruebas inmunologicas para pesquisa de virus parotiditis. herpes, enterovirus, sarampion, VIH en suero y LCR, IgM para mycoplasma: bandas oligoclonales en LCR; gases en sangre, brecha aniónica, lactacidemia, ceruloplasnina. cupremia, rastreo metabolico, aminoaciduria y aminocidemia En los electroencefalogramas (EEG), durante la segunda hospitalización, se registraba ondas bosales lentas sin actividad patoxistica $y$ el estudio de flujo sanguíneo ceretoral (Tc-HM PAO) mostrá, en la segunda semana de hospitalización. acentuada hipoperfusión global de] encéfalo. Inicialmente recibio Iraramiento sibtomático con haloperidol. que luego se cambió por L-dopa asociada con benserazjida $y$ se mantuvo un mes. Fue dado de alla con leve corea de extremidades inferiores que disminuyó paularinamente. A los 38 meses de seguimiento no habion signos de alteración neurológica.

2. Nin̄o de 7 años 10 meses de edad. previamente sano. Sin antecedentes de ingestión de fármacos o exposición a toxicos. Luego de tres semanas de cambios conductuales, jrritabilidad $e$ insomnio, presentó paroxismos de clonias hernifaciales izquierdas a las cuales luego se agregaron movimientos tónico-clónicos (TC) de extremidad superior derecha y crisis convulsivas tonico-clónicas genetalizadas. Hospitalizado durante seis semanas, en el primer dia a contar del ingreso se detectaron movimientos coreicos focales. Posteriormente se agregaron distonias faciales y orolinguales, temblor y coreoatetosis de las extremidades. dererioro oscilante de la conciencia, cambios conductuales, disartria $y$ ascitación psicomotora. Los movimientos anormales duraron en total diez semanas. No se encontraron alteraciones en es estudio citoquímico y bacseriologico en LCR; cultivo de deposiciones; pruebas inmunológicas para pesquisa de virus herpes, enterovirus, sarampión en suero y LCR; gases els sangre, brecha anionica; lactacidemia. ceruloplasmina, cupremia: rastreo metabólico: aminoaciduria aninocidemia: TAC de cerebro en la primera $y$ tercera semana de hospitalización. Las bandas oligocionales fueron negativas a la cuarta semana de hospitalización. pero positivas a lá sexta. Los EEG una semana antes $y$ una después de ingresar at hospital mostraron actividad lenta difusa e iritativa de predominio derecho. Fue tratado con haloperidol por sus movimientos anormales y -por las convulsiones (después del comienzo de los movimientos extrapiramidales)- sucesivamente con fenitoina y lucgo ácido valproico asociado a carbamazepina. Al alta estaba sin movimientos anombales, pero tenía leve disantia y bradipsiquia. que habian desaparecido siete semanas despues. El seguimiento a los 35 meses mostró indemnidad neurologica.

3. Niña de 10 años de edad, previarnente sano. Sin antecedenles de ingestión de fármacos a exposición a tóxicos. Despues de dos semanas con cefalea. mareos y comportamiento bizar to fue hospitalizado por deterioro oscilante de la conciencia, agitación psicomotora y anartria. Al tercer día de hospitalización se agregaron movimientos distónicos focales que, al cabo de otro tanto. se geberalizaron a las cuatro extremidades y eI cuelio. Posteriormente apareció temblor de las extremidades y aletosis en la superior derecha. En el LCR del día del ingreso había 10 glóbulos blancos (GB) for $\mathrm{mm}^{3}$. En la electroforesis de proteínas deI LCR, el día 10 de hospitalizacion, se encontro aumento significativo de la concentración de IgG. Los cultivos de LCR, deposiciones y pruebas inmunologicas para infecciones con virus berpes, enterovirus, sarampión. VIH y parotiditis en suero y LCR; IgM para mycoplasma; gases en sangre, brecha aniónica, lactacidemia, ceruloplasmina, cupremia: rastreo metabólico, aminoacidura y aminocidemia; TAC y RM cerebrales, dieron resultados normales o negativos. En el EEG del segundo día de hospitalización se registraba marcada desorganización lenta del hemisferio derecho $y$ de zonas anteriores $y$ temporales izquierdas. Mediante tomografía computadorizada por emisión de fotón ưnico (SPECT), el día segundo de hospitalización, se detectó. además, disminución del flujo sanguíneo pariclooccipital izquierdo. Los primeros scis días el niño recibió levodopa asociada a carbjdopa como tratamiento para los movimientos extrapiramidales anómałos, pero se les cumbió por trihexifinidilo debido a falta de respuesta. Con este último los movimientos anormales disminuyeron en forma significativa. Además, recibió dexametasona desde el tercer día de bospitatización y duranle 14 días. Fue dado de alta luego de tres semanas de hospitalización y al cabo de 26 meses no se encontraron signos de alteraciones neuroligicas.

4. Niño de 2 años 8 meses de edad, previamente sano. Sin antecedentes de ingestión de fármacos o exposición a toxicos. Después de una semana de somnolencia $y$ agitacion psicomotora. apareció corea focal de exiremidades y movirnientos distónicos faciales. Fue admitido al hospiral y durante su hospitalización se generatizaron los movimientos coreicos y se agregaron coreoaretosis, agitación psiconotora fluctuante, pérdida del lenguaje, nistagmus y crisis convulsiyas tónıco-clónicas generalizadas. Un mes después de comenzar los síntomas neurologicos tuvo varicela. Eл el LCR de la primera semana de evolución intrahospitalaria se contaron $25 \mathrm{~GB} \cdot \mathrm{mm}^{3}$ ( $90 \%$ de mononucleares y $10 \%$ de polinucleares $\rangle$, siendo normal el resto del examen citoquínico. En los EEG se registraban moderados signos lentos difusos. SPECT mostró hiperfusión cortical irregular, los nucleos de la base no se identificaron claramente. El cultivo de LCR para virus dio desartollo de enterovirus 
no polio. El resto de los exámenes, incluyendo la romografía axial y la resonancia magnetica de cerebro; cultivos para bacterias de LCR y deposiciones: pruebas inmunológicas para virus herpes. sarampion. VIH, parotiduis; gases en sangre, brecha de aniones. Jactacidemia, ceruloplasmina, cupremia, rastreos metabólicos. amino. aciduria y anjinocidemia eran nomales. Fue tratado con fenobarbital para sus crisis convulsivas idespués del inicio de los inovimientos extrapiramidales). Inicialmente recibió trihexifinidilo. que posteriormente se cambió a haloperidol y finalmente a $\mathrm{L}$-dopa. Además se le dio betametasona por dos meses desde la séptima semana de evolución. En el transcurso de seis semanas de hospitalización disminuyecon paulatinamente sos síntomas, al alta se habian recuperado ia inarcha y el lenguaje, pero persistian movimientos coceicos focales leves. Al cabo de doce meses de seguimiento no mostraba signos de secuelas neurologicas.

5. Niña de 7 años 2 meses de edad. Sin antecedentes de ingestión de fármacos o exposıción a tóxicos. Su enfermedad comenzó con labilidad emocional y lenguaje incoherente, agregándose al dfa siguiente movimientos coreicos focales de las extrejnidades $\mathrm{y}$. posieriomente. alteraciones oscilantes de la conciencla, agitación psicomotora, anartria y movimientos coreoatetósicos generalizados. Hospitalizada durante cuatro semanas, en el curso de cllas disininuyeron progresivamente sus síntomas. Fue dada de alta con sólo leves movimientos diștónicos en manos y dișartria. sin alteraciones de conciencia. En el LCR de la tercera semana habian $5 \mathrm{~GB} \cdot \mathrm{mun}^{3}$. En Jos EEG se registraba desorganizacion basal lenta difusa con elementos agudos acasionales. La TAC del cerebro era normal, pero en la RM se registraban focos pequeftos y aislodos de señal de más intensidad en T2 entre la corteza y sustancia blanca cerebelosa, sin compromiso cerebral ni de tronco. En SPECT se detectaron signos de disminución difusa del flujo sanguíneo cerebral. Inás acentuada en el hemisferio cerebeloso derecho y lisininución de flujo en el rúcleo caudado y lálamo derechos. Los exámenes restantes, incluyendo los microbiológicos para VIH y otros virus inmunológicos, nycoplasma y metah 6 licos, dieron resultados normales o negativos. Injcialmente recibió levodopa y a continuación trihexifinidilo con el que se obtuvieron mejores resultados. Dos semanas despues del alta y estando en mejores condiciones, salvo por leve disartria y muy leves movimientos distónicos en Jos dedos de ambas manos, sufrió hipectermia secundaria a una extensa neumonía aguda bilateral, choque septico secundario e hipernatremia mayor a $160 \mathrm{mEq} / \mathrm{l}$, por lo que permaneció ] 0 días en la unidad de cuidados intensiyos, seis de ellos conecrada a ventilación mecúnica, en el transcurso de la cual ocurrió un açejdente hipóxico, quedando posteriortmente con gran delerioro neurológico.

\section{Comentario}

Desde el comienzo del siglo se ha relacionado la presencia de movimientos anormales en casos de encefalitis ${ }^{5}$ y si bien es cierto esta etiología es mencionada en varios textos ${ }^{3,4}$, la comunicación de casos de movimientos anor- males y encefalitis es esporádica y prácticamente ausente en niños ${ }^{6-8}$. Lo anterior puede estar relacionado a la dificultad en sostener un diagnóstico certero de encefalitis, el cual sólo es posible establecer categoricamente mediante un examen histopatológico. La proporción de diagnóstico etiológico comunicado en distintas series de encefalitis varía entre $27 \%$ y $74 \%$-12. Los criterios indirectos (manifestaciones clínicas, exámenes funcionales como el EEG y SPECT, observación de imágenes por RM), continúan siendo los pilares fundamentales para identificar, al menos como presunción, una encefalitis. En sólo un caso de nuestra pequeña serie, a pesar de la búsqueda dirigida de agentes infecciosos conocidos, se aisló un enterovirus no polio del LCR. En los otros cuatro pacientes la falta de antecedentes de contacto con fármacos o tóxicos. los hechos clínicos, la evolución a corto y largo plazo, la exclusión de otros diagnósticos. las alteraciones registradas en EEG, SPECT -en tres de ellos-y RM -en uno-, apoyan el diagnostico de encefalitis.

Las alteraciones del movimiento han sido atribuidas a anormalidades del SNC, particularmente de los ganglios basales y sus estructuras de conexión con tálamo, corteza cerebral y cerebelo ${ }^{3}$. Distintos mecanismos etiopatogénicos han sido invocados para explicar los sintomas en enfermedades infecciosas del SNC: en las bacterianas el infarto isquémico ${ }^{13}$, la necrosis fibrinoide con trombosis de vasos meningeos basilares $^{14}$, espasmos de vasos meningeos con alteracion secundaria de flujo -de modo semejante a lo descrito en la hemorragia subaracnoidea ${ }^{15}-$ y alteraciones vasculares por hipersensibilidad retardada y depósito de inmunocomplejos ${ }^{2}$. Los mecanismos invocados en las infecciones virales del SNC han sido destrucción del tejido neural por invasión directa y multiplicación activa del virus, o reacción del tejido neural a los antígenos del agente. La destrucción neuronal se atribuye a invasión y acción directa del virus, mientras que la respuesta inmune vigotosa probablemente determina desmielinización, destrucción vascular y perivascular, y alteración secundaria de la circulación ${ }^{10}$. En tres de los pacientes que se comentan los movimientos anormales se manifestaron entre una y cuatro semanas después de comenzar otras manifestaciones clínicas de encefalitis, lo que hace pensar que en ellos, los mecanismos 
etiopatogénicos involucrados pudiesen ser de carácter autoinmune, lo cual dio base para emplear lármacos que modifican la respuesta inmune como son los corticoesteroides. Otro respaldo indirecto a la presunción de un origen básicamente autoinmunitario para este tipo de afecciones es la alteración del flujo sanguineo en el SNC, que se registró en cuatro de los pacientes descritos. El mecanismo del desarrollo de los movimientos extrapiramidales en estos pacientes es difícil de precisar, considerando que en la tomografía axial no se registraron alteraciones en ningún paciente y en la $\mathrm{RM}$ éstas se encontraron en s6lo uno de cuatro y en el cerebejo. Una posibilidad, además de la desmielinización demostrada en uno de los pacientes, es un trastorno funcional de neurotrasmisores como consecuencia de fenómenos autoinmunitarios o debidos a neurotoxinas producidas por el proceso inflamatorio o isquémico desencadenado.

La evolución clínica de los cinco pacientes estuvo, indudablemente, comandada por la aparición de movimientos anormales, constituidos principalmente por corea o atetosis y distonía, movimicntos involuntarios inicialmente focales que pronto se generalizaron. Los síntomas asociados, en especial las crisis convulsivas, la agilación psicomotriz, la falla de lenguaje, anartria y disartria, contribuyeron a dar complejidad al problema, en el diagnóstico, el tratamiento y especialmente el pronóstico.

En el diagnóstico diferencial de movimientos extrapiramidales deben considerarse varias posibilidades. Las intoxicaciones, especialmente con tranquilizantes mayores o toxicos como monóxido de carbono, pueden desencadenar movimientos anormales ${ }^{16.17}$, pero ellos son improbables en nuestros casos. Otro importante diagnóstico diferencial son los errores congénitos del metabolismo ${ }^{18}$, varios de los cuales pueden empezar con movimientos anormales: acidemia 3-metilglutacónica, aciduria glutática lipo I, déficit de sulfato oxidasa-cofactor de molibdeno, enfermedad de Lesch-Nyhan, enfermedad de Niemann-Pick tipo $\mathrm{C}$. Gml y $\mathrm{Gm} 2$, gangliosidosis de inicio tardio, keratan sulfaturia y sindrome de Leigh juvenil. Si bien es cierto no se realizaron estudios específicos para descarte de estas enfermedades, como por ejemplo determinación de ácidos orgánicos y de hexosaminidasa, el cuadro clínico, la evolución posterior y otros exámenes realizados hacen muy poco probable esta posibilidad en nuestros pacientes. El déficit de sulfato oxidasa-cofactor de molibdeno, el síndrome de Leigh juvenil y la aciduria glutárica tipo I, tienen expresiones características en la TAC y la $\mathrm{RM}$ que no se detectaron en los casos descritos. La enfermedad de Niemann-Pick tipo $\mathrm{C}$ invariablemente se presenta con parálisis de la mirada vertical y cierto grado de hepatoesplenomegalia; la keratan sulfaturia siempre se asocia a deformidades esqueléricas. Por otra parte, la evolución clínica en esie tipo de cuadros metabólicos se caracteriza por deterioro neurológico progresivo y si bien en algunos casos éste puede ser muy lento, como en ta $\mathrm{Gm} 1$, en ellos también lo es el inicio de los síntomas y éstos no remiten. Algunos casos de síndrome de Leigh de presentación juvenil pueden cursan con episodios recurrentes de movimientos extrapiramidales, con indemnidad injcial entre los episodios: sin embargo, estos pacientes pueden presentar elevación del ácido láctico plasmático y siempre presentan imágenes típicas en la RM de cerebro, consistentes en focos simétricos de alta señal en los ganglios de la base y tronco del encéfalo y secundariamente lesiones en sustancia blanca de cerebelo y cerebro. La paciente 5 fue la única que mostró anormalidades en la RM, pero éstas se limitaron al cerebelo. Por otra parte, en ella siempre se encontraron concentraciones normales de ácido láctico y su deterioro neurologico posterior estuvo claramente relacionado a un importante accidente hipóxico y a hipernatremia.

En el tratamiento sintomático de estos pacientes se intentó el uso, no sistemático, de diferentes esquemas en base a haloperidol, trihexifenidilo y levodopa. En un grupo tan pequeño de pacientes, en los cuales el uso de algunos de estos fármacos estuvo comandado por la respuesta sintomática, la cual es imposible de separar de la evolución natural de la enfermedad, es difícil obtener conclusiones definitivas, pero aparentemente el trihexifinidilo y la levodopa tendrían mejor efecto que el haloperidol en el control de estos movimientos anormales, aunque el último fármaco fue particularmente útil en el control de la agitación psicomotora, especialmente cuando fue asociado con benzodiazepinas.

E] fenobarbital, la carbamazepina y especialmente la fenitoína han sido implicados en el 
desencadenamiento de movimientos anormales ${ }^{19-23}$, sobre todo en pacientes con injuria cerebral previa ${ }^{24}$. En los dos pacientes que presentaron crisis convulsivas, los anticonvulsivos se emplearon después de aparecer los movimientos anormales, por lo que si bien es difícil reconocer su influencia sobre la evolución de los movimientos extrapiramidales, es claro que no parliciparon en su desencadenamiento. Lo anterior subraya la importancia que tiene el diagnóstico diferencial acertado entre movimientos anormales convulsivos y extrapiramidales en este tipo de pacientes.

Podemos concluir que, a pesar de ser un fenómeno infrecuente, los movimientos anormales de tipo extrapiramidal pueden presentarse en el curso de una encefalitis y que, a pesar de lo dramátzco que puede ser el cuadro clínico, las posibilidades de recuperación, en ausencia de una segunda injuria, son altamente alentadoras.

\section{Referencias}

I. Riela A. Roach ES: Choreathetosis in an infant with tuberculous meningitis. Arch Neurol 1982; 39: 596598.

2. Burtein L. Breningstall GN: Movements disorders in bacterial meningitis. J Pediatr 1986; 109: 260-264.

3. Swainth KF: Movement disorders. In: Swaiman KF. ed. Pidiatric Neurology, Principles and Practice. 2nd ed., St. Louis-Mosby-Year Book, Inc. 1994: 243-260.

4. Finfichel GM: Movemenl disorders. In: Fenichel GM. ed. Clinical Pediatric Neurology, 2nd ed. Philadelphia, W'B Saunders Co. 199.3: 285-301.

5. Marie $P$, Lery $G$ : Le syndrome excito-moteur de l'éncephalite epidemique. Rey Neurol 1920; $36 \div 513$. 536.

6. Wales's JH: Postencephalitic Parkinson syndrome after meningoencephalitis due to cossackie virus group B type 2. N Engl J Med 1960; 263: 744-746.

7. Schute DR. Buthol JS, Gutret C: Western equine encephalities with rapid onser of parkinsonism. Neurology 1977; 27: 1095-J1966.

8. Ferraz HB. Alldrade LAF: Symomatic dystonia: Clinical profile of 46 brazilian palients. Can J Neurol Sci 1992; 19: 504-501.
9. Whilley RJ, Alford CA, Hirsch $M S$, et al: Vidarabine versus acyclovir therapy in herpes simplex encephalitis. N Engl J Med 1986: 314: ]44-149.

10. Prober $C G$ : Infections of the Central Nervous System. In: Berbman RE. Kliegman RM, Arvin AM. eds. Neison Textbook of Pediatrics, 15 th ed. Philadelphia: WE Saunders Co. 1996: 707-715.

1I. Koshiniemi M. Vaheri A: Effect of measles, mumps, rubella vaccinalion on pattern of encephalitis in children. Lancet 1989; i: 31-34.

12. Koshiniemi $M$. Rautonen J, Lehtokoski-Lehtintemi $E$. Veheri A: Epidemiology of encephalitis in children: A 20 year survey. Ann Neurol 1991: 29: 492-497.

13. Udani PM. Parekh UC, Dastur DK: Neurological and related syndromes in CNS tuberculosis. J Neurol Sci 1971: 14: 341-357.

14. Polrera AA: Vascular lesions in intracraneal tuberculosis. Pathol Microbiol 1975: 43.192-194.

15. Igarashi $M$. Gilmortin RC, Gerald B, et al: Cerebral artericis and bacterial meningitis. Arch Neurol 1984; 4I: 531.535 .

16. Haddad $L M$. Winchester $J F$; Centrally active agents. In: Haddad LM, Winchester JF, eds. Clinical Management of Poisoning and Drug Oyerdose. 2nd ed. Philadelphia, WB Saunders Co, 1990: 636-892.

17. Bryson PD: Gases and abnormal hemoglobin formation. In: Bryson PD. ed. Comprehensive review in toxicology for emergency clinicians. 3rd ed. Washington DC: Taylor and Francis Co, 1996: 325-382.

18. Ljon G. Adams RD, Kolndny EH: Heredilary, predominantly extrapyramidal disorders of late childhood and adolescence. In: Lyon G, Adams RD, Kolodny EH. eds. Neurology of hereditary metabolic diseases of children. 2nd ed. New York: McGraw-Hill Co. 1996: 199.218.

19. Chadwick D. Reynolds EH. Mardsen CD: Anticonvulsant-induced dyskenesias: a comparison with dyskinesias induced by neuroleptics, J Neurol Neurosurg Psychiatry 1976; 39: 1210-1218.

20. Wiznitzer $M$. Younkin $D$ : Phenobarbital-induced dyskinesia in a neurologycally impaired child. Neurology 1984; 34: 1600-1601

21. Ahmad 5, Laidlaw J, Houghton GW, Richens A: Involuntary movements caused by phenytoin intoxication in epileptic patients. J Neurol Neurosurg Phychiatry 1975; 38: 225-231.

22. Krishnamoorthy KS, Zalneraits EL, Young RSK. Bernard $P G$; Phenytoin-induced choreathetosis in infancy: a case reports and a review. Pedianrics 1983; 72: 831.834 .

23. Luhdorf $K$. Lund $M$ : Phenytoin-induced hyperkinesia. Epilepsia 1977: 18: 409-415.

24. Crostey $C J$. Swender PT: Dystonia associated with carbamazepine administration: experience in braindamaged children. Pediatrics 1979: 63: 612-615. 